\title{
EFFECT OF SLIP CONDITION ON VISCOELASTIC MHD OSCILLATORY FORCED CONVECTION FLOW IN A VERTICAL CHANNEL WITH HEAT RADIATION
}

\author{
K.D. SINGH \\ Department of Mathematics (ICDEOL) \\ Himachal Pradesh University \\ Summer Hill, Shimla-171005, INDIA \\ E-mail: kdsinghshimla@gmail.com
}

\begin{abstract}
In this paper an oscillatory flow of a viscoelastic, incompressible and electrically conducting fluid through a porous medium bounded by two infinite vertical parallel plates is discussed. One of these plates is subjected to a slip-flow condition and the other to a no-slip condition. The pressure gradient in the channel oscillates with time. A magnetic field of uniform strength is applied in the direction perpendicular to the plates. The induced magnetic field is neglected due to the assumption of a small magnetic Reynolds number. The temperature difference of the two plates is also assumed high enough to induce heat transfer due to radiation. A closed form analytical solution to the problem is obtained. The analytical results are evaluated numerically and then presented graphically to discuss in detail the effects of different parameters entering into the problem. A number of particular cases have been shown by dotted curves in the figures. During the analysis it is found that the physical and the mathematical formulations of the problems by Makinde and Mhone (2005), Mehmood and Ali (2007), Kumar et al. (2010) and Choudhury and Das (2012) are not correct. The correct solutions to all these important oscillatory flow problems are deduced.
\end{abstract}

Key words: oscillatory, MHD, forced convection, viscoelastic, slip-flow, porous medium.

\section{Introduction}

The flow of viscoelastic fluids through porous media has attracted the attention of a large number of scholars owing to their application in the fields of extraction of energy from geothermal regions and in the flow of oil through porous rocks. Many common liquids such as certain paints, polymer solutions, some organic liquids and many new materials of industrial importance exhibit both viscous and elastic properties. The fluids with such characteristics are called viscoelastic fluids. Flows through the porous medium are also very useful in chemical engineering for the processes of purification and filtration. Rajgopal (1983) studied the heat transfer in the forced convection flow of a visco-elastic fluid of Walter's model. Singh (2011) obtained an exact solution of an oscillatory MHD flow in a channel filled with a porous medium. Rahman and Sarkar (2004) investigated the unsteady MHD flow of a viscoelastic Oldroyd fluid under time varying body forces through a rectangular channel. Singh and Singh (1966) studied an MHD flow of a dusty viscoelastic (Oldroyd B-liquid) through a porous medium between two parallel plates inclined to horizon.

Literature is now replete with the study of non-Newtonian fluid flows for different types of geometries. Rajgopal (1982) studied the flows of a non-Newtonian fluid. Rajgopal and Gupta (1984) obtained an exact solution for the flow of a non-Newtonian fluid past an infinite porous plate. Ariel (1994) analyzed the flow of a viscoelastic fluid past a porous plate. Labropulu (2000) obtained another exact solution of non-Newtonian fluid flows with prescribed vorticity. Pillai et al. (2004) studied the heat transfer in a viscoelastic boundary layer flow through a porous medium. Choudhury and Das (2000) 
investigated magnetohydrodynamic boundary layer flow of a non-Newtonian fluid past a flat plate. Metzner and White (1965) investigated the flow behavior of a viscoelastic fluid in the inlet region of a channel. Samria et al. (1990) analyzed a free convection flow of an elasto-viscous fluid past an infinite vertical plate.

The wall slip flow is another very important phenomenon that is widely encountered in this era of industrialization. It has numerous applications, for example in lubrication of mechanical devices where a thin film of lubricant is attached to the surface slipping over one another or when the surfaces are coated with special coatings to minimize the friction between them. Marques et al. (2000) considered the effect of the fluid slippage at the plate for Couette flow. Rhodes and Rouleau (1966) studied the hydrodynamic lubrication of partial porous metal bearings. The problem of the slip-flow regime is very important in this era of modern science, technology and industrialization. Hayat et al. (2008) analyzed slip flow and heat transfer of a second grade fluid past a stretching sheet through a porous space. Mehmood and Ali (2007) extended the problem of oscillatory MHD flow in a channel filled with a porous medium studied by Makinde and Mhone (2005) to slip-flow regime. Further by applying the perturbation technique Kumar et al. (2010) investigated the same problem of slip-flow regime for the unsteady MHD periodic flow of a viscous fluid through a plane channel. Very recently, Choudhury and Das (2012) studied the combined effects of the magnetic field and heat radiation on a viscoelastic flow in a channel filled with a porous medium. This study is also an extension of the paper by Makinde and Mhone (2005).

Hence, the aim of the present study is to formulate and analyze the very important physical problem of a viscoelastic, incompressible and finitely electrically conducting fluid flow through a porous medium bounded by two stationary vertical plates in the presence of heat radiation. A magnetic field of uniform strength is applied perpendicular to the plates. During the analysis it is found that there is no consistency between the physical considerations and the mathematical formulations of the problems studied by Makinde and Mhone (2005), Mehmood and Ali (2007) and Kumar et al. (2010) and the solutions obtained in all these studies are wrong. For the viscoelastic fluid flow Choudhury and Das (2012) also adopted the geometry and the boundary conditions of the problem as in Makinde and Mhone (2005) without actually verifying the validity of the governing equations for the flow considered geometrically in their Fig.1. They again repeated all the mistakes of Makinde and Mhone (2005).

\section{Mathematical analysis}

Consider the free and forced convective MHD flow of a viscoelastic, incompressible and electrically conducting fluid through a porous medium in a vertical channel. The two walls of the channel are distance ' $d$ ' apart and the temperature of one of the walls is oscillating. A Cartesian coordinate system is introduced such that the $X^{*}$-axis lies vertically upwards along the centerline of the channel. A magnetic field of uniform strength, $B_{0}$, is applied transversely along the $Y^{*}$-axis which is perpendicular to the walls of the channel. The fluid considered is finitely conducting, and hence the magnetic Reynolds number is much less than unity $(<<$ 1) so the induced magnetic field is negligible in comparison with the transversely applied magnetic field. The pressure gradient variation in the channel is also assumed to be oscillating in time. All the physical quantities are independent of $x^{*}$ for this fully developed laminar flow. The physical problem considered is presented geometrically below in Fig. 1. 


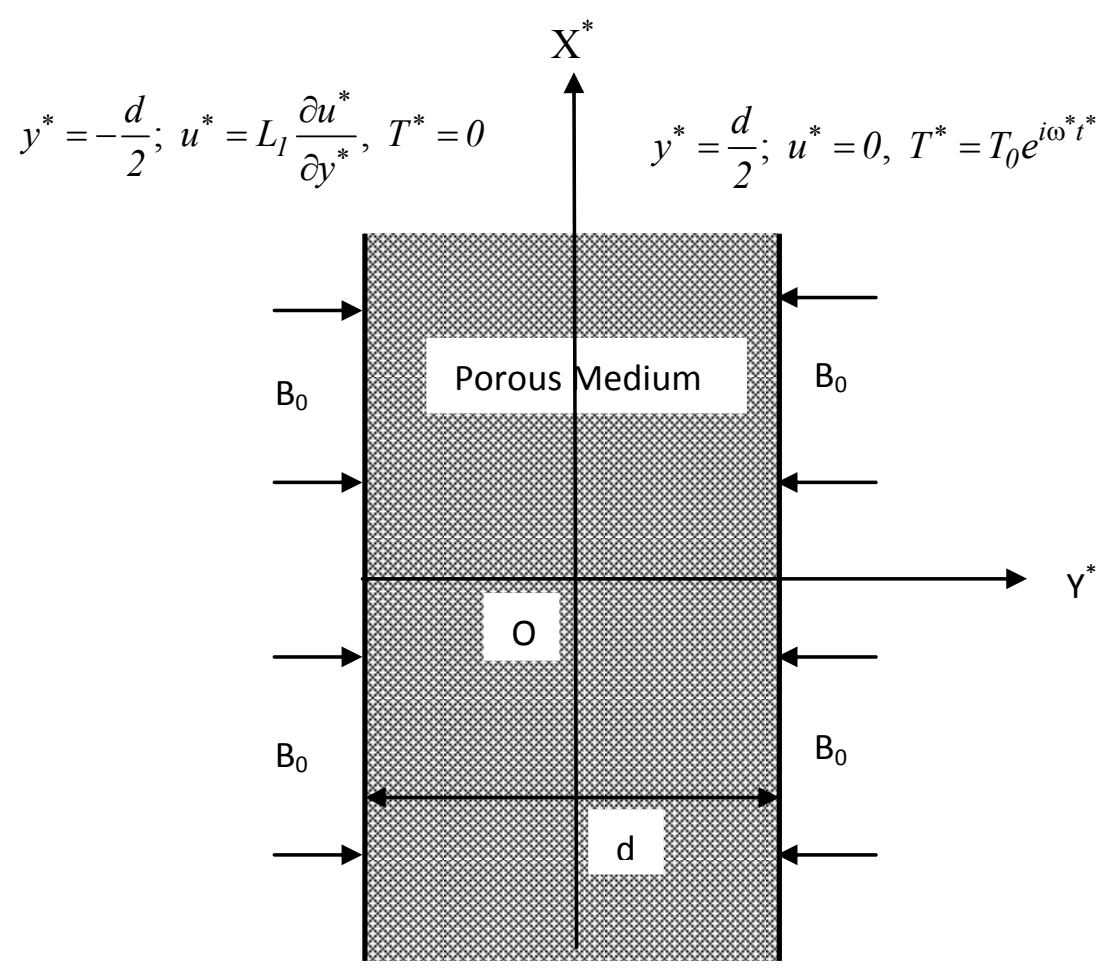

Fig.1. Geometrical presentation of the problem.

Taking into account the usual Boussinsq's approximation, the flow is governed by the following momentum and energy equations respectively

$$
\begin{aligned}
& \frac{\partial u^{*}}{\partial t^{*}}=-\frac{1}{\rho} \frac{\partial p^{*}}{\partial x^{*}}+\vartheta \frac{\partial^{2} u^{*}}{\partial y^{* 2}}+\vartheta \frac{\partial^{3} u^{*}}{\partial y^{* 2} \partial t^{*}}-\frac{\vartheta}{K^{*}} u^{*}-\frac{\sigma B_{0}{ }^{2}}{\rho} u^{*}+g \beta T^{*} \\
& \frac{\partial T^{*}}{\partial t^{*}}=\frac{k}{\rho c_{p}} \frac{\partial^{2} T^{*}}{\partial y^{* 2}}-\frac{1}{\rho c_{p}} \frac{\partial Q}{\partial y^{*}},
\end{aligned}
$$

with boundary conditions

$$
\begin{array}{ll}
u^{*}=0, & T^{*}=T_{0} e^{i \omega^{*} t^{*}} \quad \text { at } \quad y^{*}=\frac{d}{2}, \\
u^{*}=L_{l} \frac{\partial u^{*}}{\partial y^{*}}, & T^{*}=0 \quad \text { at } \quad y^{*}=-\frac{d}{2} \text { where } L_{l}=\left(\frac{2-r_{I}}{r_{I}}\right) L .
\end{array}
$$

Following Cogley et al. (1968) it is assumed that the fluid is optically thin with a relatively low density and the radiative heat flux is given by

$$
\frac{\partial Q}{\partial y^{*}}=4 \alpha^{2} T^{*}
$$


Now introducing the following non-dimensional quantities

$$
\begin{array}{ll}
x=\frac{x^{*}}{d}, & y=\frac{y^{*}}{d}, \quad u=\frac{u^{*}}{U}, \quad T=\frac{T^{*}}{T_{0}}, \\
t=\frac{t^{*} U}{d}, & \omega=\frac{\omega^{*} d}{U}, \quad p=\frac{p^{*}}{\rho U^{*}},
\end{array}
$$

in Eqs (2.1) to (2.4), we obtain equations in a dimensionless form as

$$
\begin{aligned}
& \operatorname{Re} \frac{\partial u}{\partial t}=-\operatorname{Re} \frac{\partial p}{\partial x}+(1+i \omega \gamma) \frac{\partial^{*} u}{\partial y^{2}}-\left(s^{2}+\mathrm{H}^{2}\right) u+\mathrm{Gr} T \\
& \operatorname{Pe} \frac{\partial T}{\partial t}=\frac{\partial^{2} T}{\partial y^{2}}-N^{2} T
\end{aligned}
$$

with boundary conditions

$$
\begin{aligned}
& u=0, \quad T=e^{i \omega t}, \quad \text { at } \quad y=\frac{1}{2}, \\
& u=h \frac{\partial u}{\partial y}, \quad T=0, \quad \text { at } \quad y=-\frac{1}{2}
\end{aligned}
$$

where

$\operatorname{Gr}($ Grashof number $)=\frac{g \beta T_{0} d^{2}}{\vartheta U}$,

$\gamma($ viscoelastic parameter $)=\frac{\vartheta_{1} \operatorname{Re}}{d^{2}}$,

Pe $($ Peclet number $)=\frac{\rho c_{p} U d}{k}$,

$\operatorname{Re}($ Reynolds number $)=\frac{U d}{\vartheta}$,

$\mathrm{H}($ Hartmann number $)=B_{0} d \sqrt{\frac{\sigma}{\vartheta}}$,

Da (Darcy number $)=\frac{K^{*}}{d^{*}}$, 


$$
\begin{aligned}
& s \text { (porous medium shape factor parameter })=\frac{1}{\sqrt{\mathrm{Da}}}, \\
& N(\text { radiation parameter })=2 \alpha \frac{d}{\sqrt{k}}, \\
& h(\text { slip-flow parameter })=\frac{L_{1}}{d} .
\end{aligned}
$$

For the oscillatory internal flow in the channel, the oscillatory pressure gradient is assumed to be of the form $-\frac{\partial p}{\partial x}=\mathrm{Pe}^{i \omega t}$.

\section{Method of solution}

In order to solve Eqs (2.7) and (2.8) under the boundary conditions Eqs (2.9) and (2.10), let us assume for a purely oscillatory flow

$$
u(y, t)=u_{0}(y) e^{i \omega t}, \quad T(y, t)=\theta_{0}(y) e^{i \omega t} \quad \text { and } \quad-\frac{\partial p}{\partial x}=\mathrm{Pe}^{i \omega t} .
$$

Substituting these expressions in Eqs (2.7) to (2.10), we obtain

$$
\begin{aligned}
& l^{2} \frac{d^{2} u_{0}}{d y^{2}}-m^{2} u_{0}=-P \mathrm{Re}-\mathrm{Gr} \theta_{0} \\
& \frac{d^{2} \theta_{0}}{d y^{2}}-n^{2} \theta_{0}=0
\end{aligned}
$$

where $l=\sqrt{1+i \omega \gamma}, \quad m=\sqrt{\left(s^{2}+\mathrm{H}^{2}+i \omega \mathrm{Re}\right)}$ and $n=\sqrt{\left(N^{2}+i \omega \mathrm{Pe}\right)}$ and with transformed boundary conditions

$$
\begin{aligned}
& u_{0}=0, \quad \theta_{0}=1 \quad \text { at } \quad y=\frac{1}{2} \text {, } \\
& u_{0}=h \frac{\partial u_{0}}{\partial y}, \quad \theta_{0}=0 \quad \text { at } \quad y=-\frac{1}{2} \text {. }
\end{aligned}
$$

The ordinary differential equations (2.12) and (2.13) are solved under boundary conditions (2.14) and (2.15) and the solutions for the velocity and the temperature fields are obtained, respectively, as 


$$
\begin{aligned}
& u(y, t)=\left[\begin{array}{l}
\left.\frac{P \operatorname{Re}}{m^{2}}\left\{\frac{2 \sinh \frac{m}{2 l} \cosh \frac{m}{l} y+\frac{m}{l} h \cosh \frac{m}{l}\left(y+\frac{l}{2}\right)}{\left(\sinh \frac{m}{l}+\frac{m}{l} h \cosh \frac{m}{l}\right)}\right\}+\frac{\mathrm{Gr}}{\left(l^{2} n^{2}-m^{2}\right) \sinh n}\right] \\
\left\{\frac{\sinh n\left[\sinh \frac{m}{l}\left(y+\frac{l}{2}\right)+\frac{m}{l} h \cosh \frac{m}{l}\left(y+\frac{l}{2}\right)\right]+n h \sinh \frac{m}{l}\left(y-\frac{1}{2}\right)}{\left(\sinh \frac{m}{l}+\frac{m}{l} h \cosh \frac{m}{l}\right)-\sinh n\left(y+\frac{l}{2}\right)}\right\}
\end{array}\right] e^{i \omega t},(2.1) \\
& T(y, t)=\frac{\sinh n\left(y+\frac{1}{2}\right)}{\sinh n} e^{i \omega t} .
\end{aligned}
$$

For $\gamma=0$, i.e., for the case of a Newtonian fluid the physical problem reduces to the one studied by Mehmood and Ali (2007) and its correct solutions for the temperature and the velocity fields are given by Eq.(2.17) and Eq.(2.18) below

$$
u(y, t)=\left[\begin{array}{l}
\frac{P \operatorname{Re}}{m^{2}}\left\{1-\frac{2 \sinh \frac{m}{2} \cosh m y+m h \cosh m\left(y+\frac{1}{2}\right)}{(\sinh m+m h \cosh m)}\right\}+\frac{\mathrm{Gr}}{\left(n^{2}-m^{2}\right) \sinh n} \\
\left\{\frac{\sinh n\left[\sinh m\left(y+\frac{1}{2}\right)+m h \cosh m\left(y+\frac{1}{2}\right)\right]+n h \sinh m\left(y-\frac{1}{2}\right)}{(\sinh m+m h \cosh m)-\sinh n\left(y+\frac{1}{2}\right)}\right\}
\end{array}\right] e^{i \omega t} .
$$

The solution of the problem studied by Choudhury and Das (2012) can also be obtained by taking $h=0$, i.e., in the absence of the slip-flow condition, in Eq.(2.16). By taking $\gamma=0$ and $h=0$ in Eq.(2.16) the correct form of the solution of the physical problem studied by Makinde and Mhone (2005) is obtained as

$$
u(y, t)=\left[\frac{P \operatorname{Re}}{m^{2}}\left\{1-\frac{\cosh m y}{\cosh \frac{m}{2}}\right\}+\frac{\mathrm{Gr}}{\left(n^{2}-m^{2}\right)}\left\{\frac{\sinh m\left(y+\frac{1}{2}\right)}{\sinh m}-\frac{\sinh n\left(y+\frac{1}{2}\right)}{\sinh n}\right\}\right] e^{i \omega t} .
$$

However, the solution of the energy equation for the temperature field $T(y, t)$ remains the same. The following limiting cases prove the validity and correctness of the analysis:

(i) For $\mathrm{Gr}=h=\gamma=0$, i.e., in the absence of convection currents, the slip-flow condition and for the viscous fluid in the horizontal channel the solution is already obtained by Singh and Rina (2010).

(ii) Further by taking $s=\mathrm{H}=0$, i.e., treating the medium in the channel as ordinary and in the absence of transverse magnetic field the solution reduces to 


$$
u(y, t)=\frac{P}{i \omega}\left(1-\frac{\cosh \sqrt{i \omega \mathrm{Re} y}}{\cosh \frac{\sqrt{i \omega \mathrm{Re}}}{2}}\right) e^{i \omega t}
$$

This is the dimensionless form of the very well known result reported by Schlichting and Gersten (2001) (pp.137).

From the velocity field in Eq.(2.16) we can obtained the skin-friction at the left wall in terms of its amplitude $|F|$ and the phase angle $\varphi$ as

$$
\tau_{L}=|F| \cos (\omega t+\varphi)
$$

where

$$
\begin{aligned}
& F_{r}+i F_{i}=\frac{A \operatorname{Re}}{\operatorname{lm}}\left\{\frac{\cosh \frac{m}{l}-1}{\left(\sinh \frac{m}{l}+\frac{m}{l} h \cosh \frac{m}{l}\right)}\right\}+ \\
& +\frac{\mathrm{Gr}}{\left(n^{2}-m^{2}\right) \sinh n}\left\{\frac{m}{l}\left(\frac{\sinh n+n h \cosh \frac{m}{l}}{\sinh \frac{m}{l}+\frac{m}{l} h \cosh \frac{m}{l}}\right)-n\right\} .
\end{aligned}
$$

The amplitude and the phase angle are respectively given by

$$
|F|=\sqrt{F_{r}^{2}+F_{i}^{2}} \quad \text { and } \quad \varphi=\tan ^{-1} \frac{F_{i}}{F_{r}} .
$$

From the temperature field the rate of heat transfer $\mathrm{Nu}$ (Nusselt number) in terms of its amplitude and the phase angle can be obtained as

$$
\mathrm{Nu}=\left(\frac{\partial T}{\partial \eta}\right)_{\eta=-\frac{1}{2}}=|\mathrm{H}| \cos (\omega t+\psi) \quad \text { where } \quad \mathrm{H}_{r}+i \mathrm{H}_{i}=\frac{n}{\sinh n}
$$

The amplitude $|\mathrm{H}|$ and the phase angle $\psi$ of the rate of heat transfer are respectively given by

$$
|\mathrm{H}|=\sqrt{\left(\mathrm{H}_{r}^{2}+\mathrm{H}_{i}^{2}\right)} \quad \text { and } \quad \psi=\tan ^{-1} \frac{\mathrm{H}_{i}}{\mathrm{H}_{r}} .
$$

\section{Results and discussion}

The problem of the oscillatory convective and radiative MHD flow of a viscoelastic fluid through a porous medium in a vertical channel is analyzed under slip-flow conditions. The closed form solutions for 
the velocity and temperature fields are obtained and then evaluated for various values of different parameters present in the equations. To have a better insight into the physical problem the variations of the physical quantities with flow parameters are shown graphically.

Figure 2 shows the variation of the velocity with the viscoelastic parameter $(\gamma)$, the slip-flow parameter $(h)$, Reynolds number $(\mathrm{Re})$, Hartmann number $(\mathrm{H})$, porous medium shape factor $(s)$, pressure gradient $(A)$ and the frequency of oscillations $(\omega)$. The dotted $(\ldots$.$) and dashed (----) curves, respectively,$ represent the cases of a Newtonian fluid $(\gamma=0)$ with no-slip condition $(h=0)$ and slip condition $h \neq 0$. It is observed that the velocity decreases with the increase of $\gamma$, i.e., the flow retards as the viscoelastic parameter increases (curves I and II). As is expected velocity increases with the increase of the slip flow parameter $(h)$.

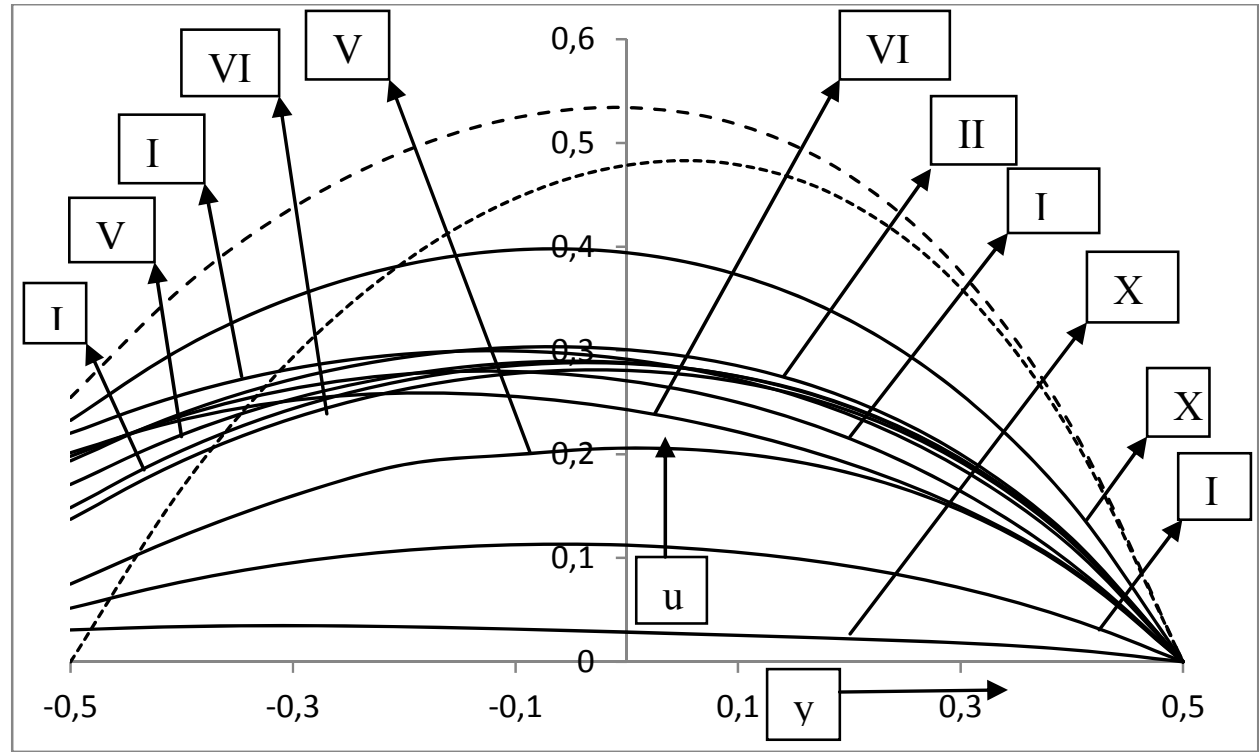

Fig.2. Velocity profiles for $t=0$.

Table 1.The curves in Fig.2 represent the values of parameters given in this table.

\begin{tabular}{|c|c|c|c|c|c|c|c|c|}
\hline$\gamma$ & $h$ & $\mathrm{Gr}$ & $\mathrm{Re}$ & $\mathrm{H}$ & $S$ & $\operatorname{Pe} N$ & $P$ & \\
\hline 0 & 0 & 5 & 1 & 2 & 1 & 11 & 5 & $5 \ldots$ \\
\hline 0 & 0.5 & 5 & 1 & 2 & 1 & 11 & 5 & 5 ---- \\
\hline 0.2 & 0.5 & 5 & 1 & 2 & 1 & 11 & 5 & $5 I$ \\
\hline 0.5 & 0.5 & 5 & 1 & 2 & 1 & 11 & 5 & $5 I I$ \\
\hline 0.2 & 1.0 & 5 & 1 & 2 & 1 & 11 & 5 & $5 I I I$ \\
\hline 0.2 & 0.5 & 1 & 1 & 2 & 1 & 11 & 5 & $5 \mathrm{IV}$ \\
\hline 0.2 & 0.5 & 5 & 0.5 & 2 & 1 & 11 & 5 & $5 \mathrm{~V}$ \\
\hline 0.2 & 0.5 & 5 & 1 & 3 & 1 & 11 & & $5 V I$ \\
\hline 0.2 & 0.5 & 5 & 1 & 2 & 0.5 & 11 & 5 & 5 VII \\
\hline 0.2 & 0.5 & 5 & 1 & 2 & 1 & $\begin{array}{ll}7 & 1\end{array}$ & 5 & 5 VIII \\
\hline 0.2 & 0.5 & 5 & 1 & 2 & 1 & 15 & 5 & $5 I X$ \\
\hline 0.2 & 0.5 & 5 & 1 & 2 & 1 & 11 & 7 & $5 X$ \\
\hline 0.2 & 0.5 & 5 & 1 & 2 & $\begin{array}{ll}1 & 1\end{array}$ & 15 & & $5 X I$ \\
\hline
\end{tabular}


An increase in the velocity is also noticed with an increase of the Reynolds number Re, Hartmann number $\mathrm{H}$, porous medium shape factor parameter $s$, and the pressure gradient $P$. The increasing Reynolds number means (being the ratio of inertial to viscous forces) that inertial forces are predominant and strengthen the velocity field further. The increasing Hartmann number means that the increasing transverse magnetic field strength induces a drag force to the retarding flow due to the viscoelastic fluid. Similarly, a more favorable pressure gradient leads to a faster flow. It is observed that the velocity field decreases in the left half of the channel but increases in the right half due to the increase in the Grashof number. Similarly, the increase in the Peclet number and the radiation parameter leads to an increase in the left half but to a decrease in the right half of the channel. The velocity profiles also decrease with increasing frequency of oscillations $\omega$.

The numerical data for the amplitude $|F|$ and the phase angle $\varphi$ of the skin-friction $\tau_{L}$ at the plate $(y$ $=-0.5)$ with the slip-flow condition are shown in Figs 3 and 4 respectively. The effect of each parameter is ascertained through the curves keeping rest of the parameters fixed. In Fig. 3 the dotted curve represents the amplitude for the case of a Newtonian fluid and which is maximum. Curves, I and II show clearly that the skin-friction amplitude decreases with the increase of the viscoelastic parameter $\gamma$. The amplitude $|F|$ of the skin-friction also decreases with the increase of the slip-flow parameter $h$ (Curves I and III). This is because of the fact that the frictional force is reduced substantially due the presence of a thin layer of lubricant on the surface. The increase of the Grashof number Gr (Curves I and IV), Reynolds number Re (Curves I and V) or the porous medium shape factor $s$ (Curves I and VII) leads to the increases of amplitude $|F|$ of the skinfriction. This figure clearly shows that $|F|$ decreases with the increasing magnetic field strength parameter, the Hartmann number H (Curves I and VI). The Peclet number Pe (Curves I and VIII) and the radiation parameter $N$ (Curves I and IX) lead to a decrease in the skin-friction amplitude $|F|$. The amplitude $|F|$ goes on decreasing with increasing values of the frequency of oscillations, $\omega$.

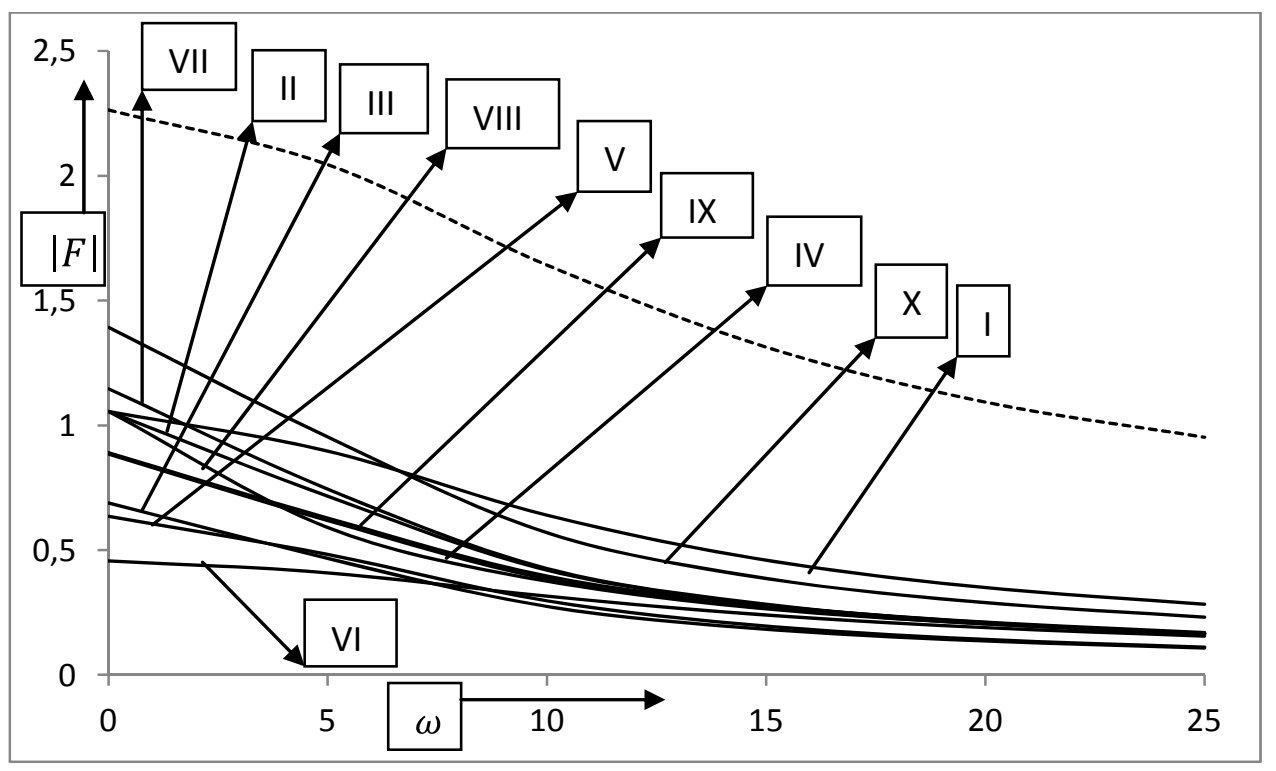

Fig.3. Amplitude of the skin friction. 


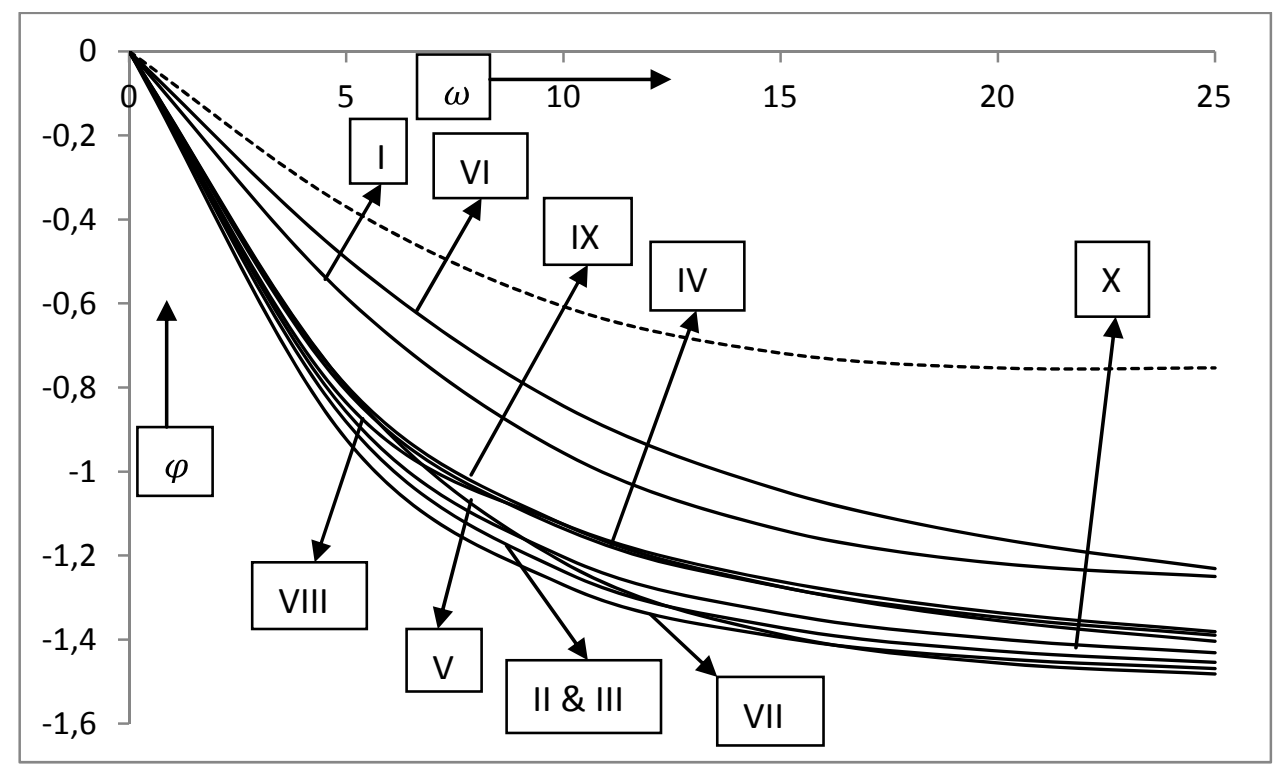

Fig.4. Phase of the skin friction.

Table 2.The curves in Figs 3 and 4 represent the values of parameters given in this table.

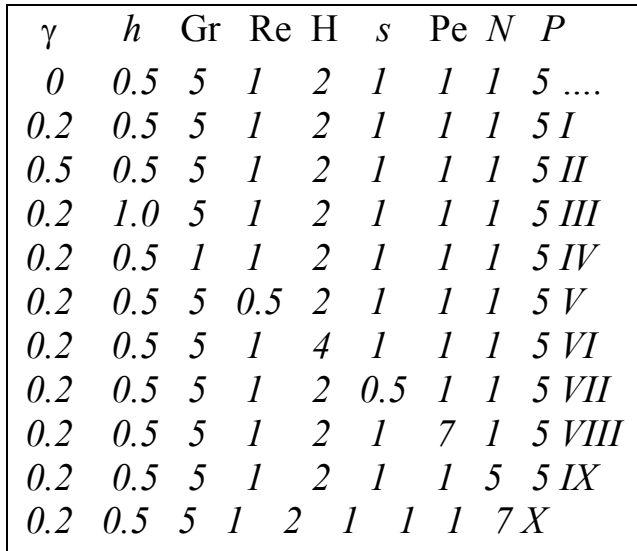

The phase angle $\varphi$ of the skin-friction $\tau_{L}$ is plotted against $\omega$ in Fig.4. Since all the curves in this figure are for the negative values of $\varphi$, it means that there is always a phase lag and this phase lag goes on decreasing further with increasing frequency of oscillations $\omega$. The increase in the phase lag is quite significant with the increase of the viscoelastic parameter $\gamma$ (Curves I and II), the slip-flow parameter $h$ (Curves I and III) and the Hartmann number H (Curves I and VI) and pressure gradient $A$ (Curves I and X). However, the phase lag decreases due to the increase of the Grashof number Gr (Curves I and IV), the Reynolds number Re (Curves I and V), the porous medium shape factor parameter $s$ (Curves I and VII), the Peclet number Pe (Curves I and VIII) and the radiation parameter $N$ (Curves I and IX).

\section{Nomenclature}

$$
\begin{aligned}
& B-\text { magnetic field applied } \\
& c_{p} \text { - specific heat at constant pressure } \\
& \text { Da - Darcy number } \\
& |F| \text { - amplitude of skin friction }
\end{aligned}
$$




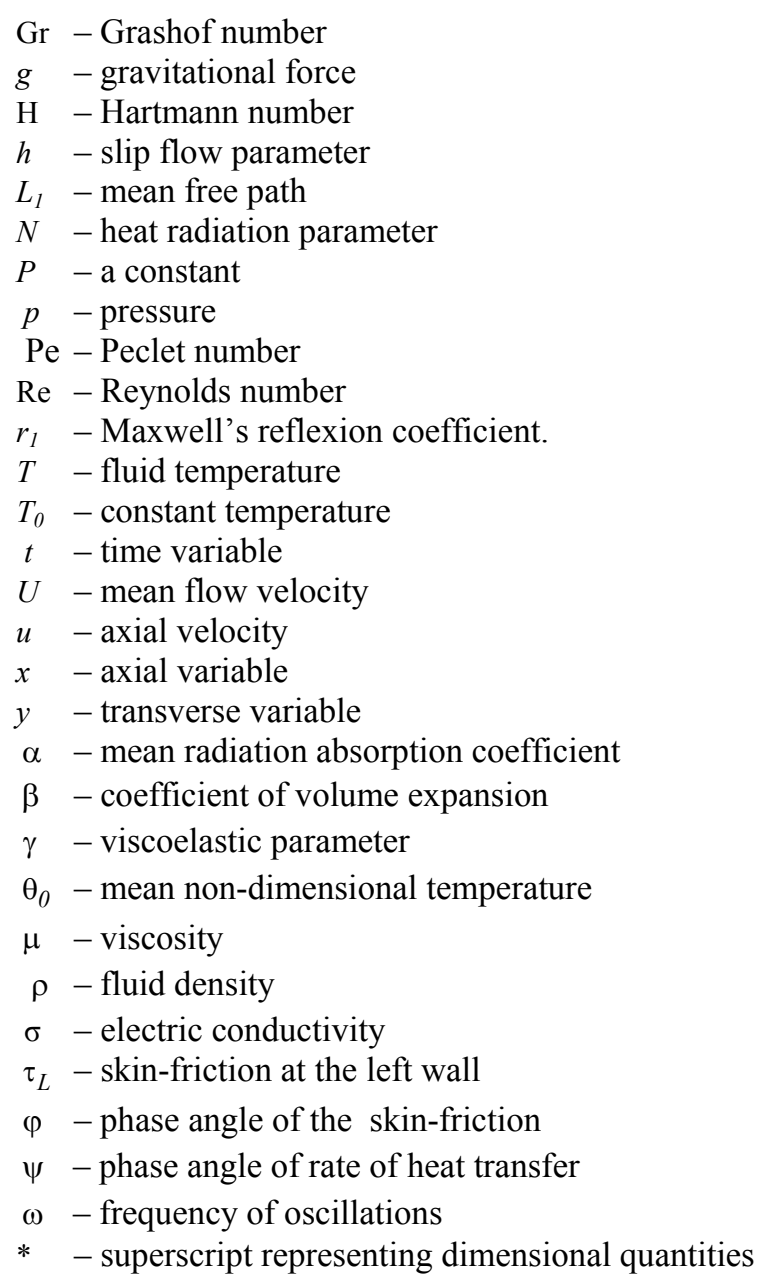

\section{References}

Ariel P.D. (1994): The flow of a viscoelastic fluid past a porous plate. - Acta Mech., vol.107, pp.199-204.

Choudhory R. and Das A. (2000): Magnetohydrodynamics boundary layer flow of non-Newtonian fluid past a flat plate. - Int. J. Pure and Appl. Math., vol.31, pp.1429-1441.

Choudhory R. and Das U.J. (2012): Heat transfer to MHD oscillatory viscoelastic flow in a channel filled with porous medium. - Physics Research International.

Cogley A.C., Vincenti W.G. and Gill S.E. (1968): Differential approximation for radiative transfer in a non-gray-gas near equilibrium. - AIAA. J. vol.6, pp.551-553.

Hayat T., Javed T. and Abbas Z. (2008): Slip flow and heat transfer of a second grade fluid past a stretching sheet through a porous space. - Int. J. Heat Mass Transfer, vol.51, pp.4528-4534.

Kumar A., Varshney C.L. and Sajjan L. (2010): Perturbation technique to unsteady MHD periodic flow of viscous fluid through a planner channel. - J. of Engng. and Tech. Research, vol.2, pp.73-81.

Labropulu F. (2000): Exact solution of non-Newtonian fluid flows with prescribed vorticity. - Acta Mech., vol.141, pp.11-20.

Makinde O.D. and Mhone P.Y. (2005): Heat transfer to MHD oscillatory flow in a channel filled with porous medium. - Rom. Journ. Phys., vol.50, pp.931-938.

Mehmood A. and Ali A. (2007): The effect of slip condition on unsteady MHD oscillatory flow of a viscous fluid in a planer channel. - Rom. Journ. Phys., vol.52, pp. 85-91. 
Mentzer A.B. and White J.L. (1965): Flow behavior of viscoelastic fluids in the inlet region of a channel. - AlChem E J., vol.11, pp.989-995.

Morques Jr.W., Kermer G.M. and Shapiro F.M. (2000): Couette flow with slip and jump boundary conditions. Continuum Mech. Therodynam., vol.12, pp.379-386.

Pillai K.M.C., Sai K.S., Swamy N.S., Nataraja H.R., Tiwari S.B. and Rao B.N. (2004): Heat transfer in a viscoelastic boundary layer flow through porous medium. - Comput. Mech., vol.34, pp.27-37.

Rahmann M.M. and Sarkar M.S.A. (2004): Unsteady MHD flow of viscoelastic Oldroyd fluid under time varying body forces through a rectangular channel. - Bulletin of Calcutta Mathematical Society, vol.96, pp.463-470.

Rajgopal K.R. (1982): A note on unsteady unidirectional flows of a non-Newtonian fluid. - Int. J. Non-Linear Mech., vol.17, pp.369-373.

Rajgopal K.R. (1984): On the creeping flow of a second order fluid. - J. Non-Newtonian Fluid Mech., vol.15, pp.239246.

Rajgopal K.R. and Gupta A.S. (1984): An exact solution for the flow of a non-Newtonian fluid past an infinite porous plate. - Meccanica, vol.19, pp.158-160.

Rhodes C.A. and Rouleau W.T. (1966): Hydromagnetic lubrication of partial metal bearings. - J. Basic Eng. T. ASME, vol.88, pp.53-60.

Samria N.K., Prasad R. and Reddy M.U.S. (1990): MHD free convection flow of an elasto-viscous fluid past an infinite vertical plate. - Astro. Physics and Space Science, vol.181, pp.125-134.

Schlichting H. and Gersten K. (2001): Boundary Layer Theory. - Springer-Verlag, $8^{\text {th }}$ edition, pp137.

Singh A.K. and Singh N.P. (1966): MHD flow of a dusty viscoelastic liquid through a porous medium between two inclined parallel plates. - Proc. of National Academy of Sciences India, vol.66A, pp.143-150.

Singh K.D. (2011): Exact solution of an oscillatory MHD flow in a channel filled with porous medium. - Int. J. Applied Mechanics and Engineering, vol.16, pp.277-283.

Singh K.D. and Devi R. (2010): Effect of slip velocity on MHD oscillatory flow through porous medium in a channel. International Journal of Physics, vol.3, pp.75-83.

Received: May 17, 2012

Revised: August 18, 2012 